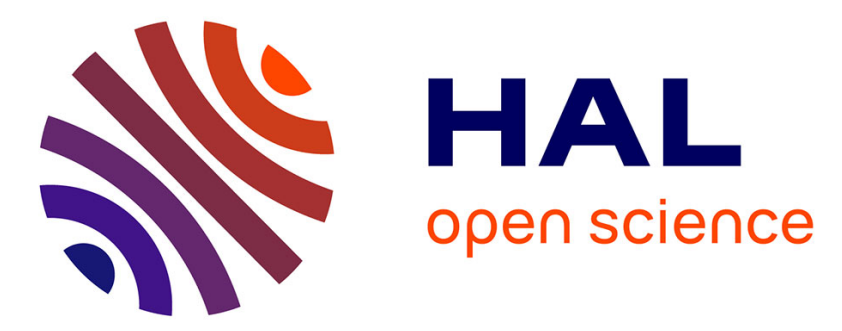

\title{
Influence of the scale of fluctuation of the friction angle on the face stability of a pressurized tunnel in sands
} Guilhem Mollon, Kok-Kwang Phoon, Daniel Dias, Abdul-Hamid Soubra

\section{To cite this version:}

Guilhem Mollon, Kok-Kwang Phoon, Daniel Dias, Abdul-Hamid Soubra. Influence of the scale of fluctuation of the friction angle on the face stability of a pressurized tunnel in sands. ASCE Georisk 2011: Geotechnical Risk Assessment and Management, 2011, Atlanta, United States. 10.1061/41183(418)14 . hal-01008337

\section{HAL Id: hal-01008337 \\ https://hal.science/hal-01008337}

Submitted on 29 Apr 2018

HAL is a multi-disciplinary open access archive for the deposit and dissemination of scientific research documents, whether they are published or not. The documents may come from teaching and research institutions in France or abroad, or from public or private research centers.
L'archive ouverte pluridisciplinaire HAL, est destinée au dépôt et à la diffusion de documents scientifiques de niveau recherche, publiés ou non, émanant des établissements d'enseignement et de recherche français ou étrangers, des laboratoires publics ou privés. 


\section{Influence of the Scale of Fluctuation of the Friction Angle on the Face Stability of a Pressurized Tunnel in Sands}

M. Guilhem Mollon ${ }^{1}$, Kok-Kwang Phoon ${ }^{2}$, F.ASCE, Daniel Dias ${ }^{3}$, and Abdul-Hamid Soubra $^{4}$, M.ASCE

${ }^{1}$ LGCIE, INSA Lyon, Université de Lyon, Domaine scientifique de la Doua, Bât JCA Coulomb, 20 Avenue Albert Einstein, 69621 Villeurbanne, France ; guilhem.mollon@insa-lyon.fr

${ }^{2}$ Civil Engineering Department, National University of Singapore, Singapore; Block E1A, \#07-03, 1 Engineering Drive 2, Singapore 117576; cvepkk@nus.edu.sg

${ }^{3}$ LGCIE, INSA Lyon, Université de Lyon, Domaine scientifique de la Doua, Bât JCA Coulomb, 20 Avenue Albert EINSTEIN, 69621 Villeurbanne, France ; daniel.dias@insa-lyon.fr

${ }^{4}$ GeM, Université de Nantes, Boulevard de l'université, BP 152, 44603 Saint-Nazaire cedex, France ; abed.soubra@univ-nantes.fr

\section{ABSTRACT}

The present paper makes use of a 2D limit analysis mechanism developped in Mollon et al. 2011 to study the influence of the scale of fluctuation of the friction angle in a soil mass on the critical collapse pressure of a pressurized tunnel face. The variability of the soil is simulated by random fields in the framework of the Karhunen-Loeve Expansion method, with various scales of fluctuation. A MonteCarlo sampling scheme is used to examine the probabilistic impact of this variability as well as possible emergent behaviours that may be qualitatively different from well known behaviours associated with homogeneous sand. Probabilistic results show that $\mathrm{L} / \mathrm{D}$ ratio (i.e. autocorrelation length of the friction angle random field, over tunnel diameter D) has an important influence on the dispersion of the critical collapse pressure. For a small to moderate value of this scale of fluctuation with respect to the tunnel diameter, it appears that there are several critical positions of the failure mechanism, whereas such phenomenon is unlikely for large values of $\mathrm{L} / \mathrm{D}$.

\section{INTRODUCTION}

The estimation of the fluid pressure that has to be applied to a tunnel face to maintain its stability is of major interest in real tunnelling projects. This paper aims at investigating the impact of the scale of fluctuation of the friction angle of sands on the collapse of a pressurized tunnel face. This is done by using a $2 \mathrm{D}$ analytical collapse mechanism defined in the framework of the kinematical theorem of limit analysis. This mechanism was extensively presented and validated in homogeneous and heterogeneous sands in a previous study (Mollon et al. 2011), and appeared to provide a reasonable estimate of the critical collapse pressure and of the shape of failure in a spatially varying sand, at a moderate time cost. Extensive stochastic simulations of heterogeneous sands are used herein to evaluate the role of the scale of fluctuation of the soil friction angle and its impact on the face stability.

The collapse mechanism is a rotational rigid block as shown in Fig. 1. It moves with a cylindrical velocity field about point $\mathrm{O}$. The geometrical construction of this mechanism is based on a spatial discretization of a lower and an upper slip lines, emerging respectively from two points $\mathrm{A}$ and $\mathrm{B}$ belonging to the tunnel face. The 
failure mechanism is defined by four geometrical parameters as shown in Fig. 1a: $\mathrm{R}$ and $\beta$ are related to point $O$ (centre of rotation), and $H$ and $R_{m}$ are related to the position of the two points $\mathrm{A}$ and $\mathrm{B}$. The discretization process (Fig. 1b) uses a large number of radial lines meeting in $O$ (two successive lines are separated by angle $\delta \alpha$ ). Thus, the two slip lines $\mathrm{AE}$ and $\mathrm{BE}$ are divided into a large number of small segments, which respect locally the normality condition related to the kinematical theorem (i.e. each segment of the slip lines makes an angle $\varphi$ with the velocity vector, $\varphi$ being dependant on the coordinates ( $\mathrm{x}, \mathrm{y})$ of the point considered). The general assumptions of this mechanism and its generation are detailed in Mollon et al. [2011]. For each set of the four geometrical parameters, the kinematical theorem of limit analysis allows one to obtain a value of the collapse pressure $\sigma_{\mathrm{c}}$. The optimal value of this pressure is obtained by maximization with respect to the four geometrical parameters. A tunnel with a diameter $\mathrm{D}=10 \mathrm{~m}$ is adopted as an illustrative example in this study along with a soil unit weight equal to $18 \mathrm{kN} / \mathrm{m}^{3}$.

a.

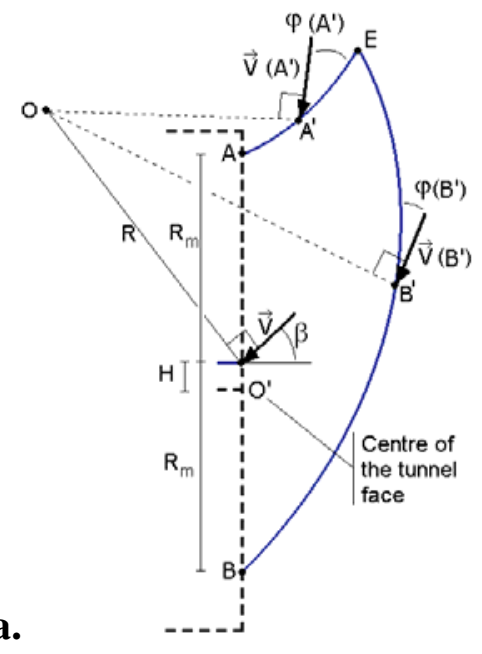

b.

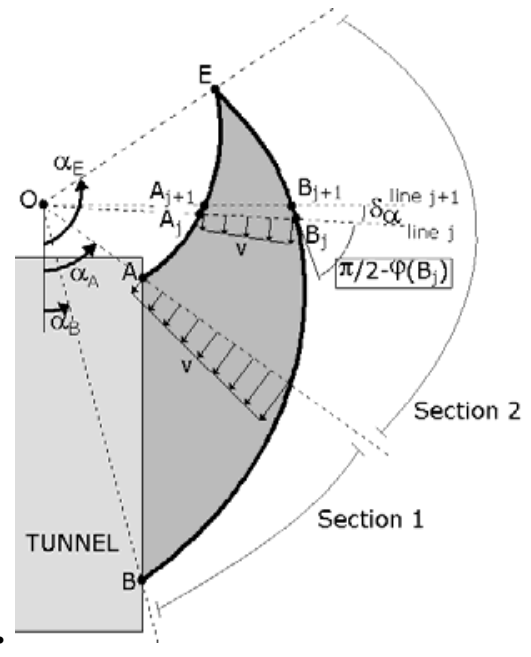

Figure 1. a. General description of the collapse mechanism; b. Discretization scheme

\section{SIMULATION OF RANDOM FIELDS}

The spatial variability of the sand is modelled here by random fields, which are simulated using the well known Karhunen-Loeve (KL) expansion. In this preliminary attempt to investigate the impact of the spatial variability of the friction angle on the collapse of a tunnel face, only two-dimensional lognormal fields with a mean $\mu_{\log }=30^{\circ}$ and a standard deviation $\sigma_{\log }=3^{\circ}$ (i.e. a coefficient of variation of $10 \%$ ) are used. The simulation of such a field requires an underlying normal field with a mean $\mu_{\text {normal }}$ and a standard deviation $\sigma_{\text {normal }}$ which are deduced from the target mean and standard deviation $\mu_{\log }$ and $\sigma_{\log }$ (Sudret and Der Kiureghian [2000]). An exponential autocorrelation function is chosen for this underlying normal field:

$$
\rho(A, B)=\exp \left(-\frac{\left|x_{A}-x_{B}\right|}{L_{x}}-\frac{\left|y_{A}-y_{B}\right|}{L_{y}}\right)
$$


It can be demonstrated that, as long as the COV remains low (which is true in the present case), the autocorrelation function of the lognormal field is not far from the one of the underlying field. The detailed method of simulating the underlying normal field can be found elsewhere (Sudret and Der Kiureghian [2000], Phoon et al. [2005]). Only isotropic random fields with $\mathrm{L}_{\mathrm{x}}=\mathrm{L}_{\mathrm{y}}=\mathrm{L}$ are used in this study. Several $\mathrm{L} / \mathrm{D}$ values are studied here, as well as the two limit cases $\mathrm{L} / \mathrm{D}=0$ and $\mathrm{L} / \mathrm{D}=\infty$. An infinite autocorrelation length corresponds to a homogeneous field, and can be modelled by only one random variable. On the other hand, an autocorrelation length equal to zero corresponds to a non-correlated field, i.e. a soil in which the value of $\varphi$ at each point is totally uncorrelated with the ones of surrounding points. Notice that the KL expansion is not able to generate such a field, and another approach is used here. The soil mass is divided into a very large number of square pixels with a size of $1 \mathrm{~cm}$ (i.e. $\mathrm{D} / 1000$ for a tunnel diameter of $10 \mathrm{~m}$ ), and each pixel is assigned a random value of $\varphi$ respecting the lognormal distribution given previously. This process is believed to provide a reasonable approximation to an uncorrelated field.

\section{RESPONSE SURFACES}

The response surfaces obtained for some realizations of the random field corresponding to various L/D values are provided in Fig. 2. These surfaces are represented by lines of equal value of the collapse pressure $\sigma_{c}$ in the plane of the two geometrical parameters $\beta$ and $\mathrm{R}$ of the mechanism (note that, for this figure, a full face failure was considered, i.e. $H=0 m$ and $R_{m}=5 m$ ). The solid lines denote an interval of $1 \mathrm{kPa}$. The dotted lines denote a more refined interval of $0.2 \mathrm{kPa}$. The most probable collapse mechanism is the one for which the value of $\sigma_{\mathrm{c}}$ is maximal. For homogeneous fields $(L / D=\infty)$, Fig. 2 shows that the response surface is very regular and that the maximum is unique. It can therefore be found very easily with a classical optimisation tool. In heterogeneous soils, however, the response surface is much more complex. In the case $L / D=1$, its shape is slightly modified and leads to two very close local maximums, which correspond to two collapse mechanisms with a quasisimilar shape. When $L / D=0.1$, the response surface becomes much more distorted, and leads to two different maximums with very different values of the parameters $\beta$ and R. However, one of these two maximums has a much larger value of $\sigma_{\mathrm{c}}$ and probably leads to the preferential failure mechanism. For the two cases $L / D=1$ and $\mathrm{L} / \mathrm{D}=0.1$, the surface remains quite smooth, and optimisation algorithms are able to find the global maximum without difficulty. On the contrary, the case $L / D=0$ shows a very complex response surface, with a large number of local maximums. Optimisation algorithms are not able to provide the exact location of the global maximum of such a surface, but provide a correct estimate of the maximal value of $\sigma_{\mathrm{c}}$ because this surface is quite flat at its top. In other words, there are a large number of fairly distinctively different mechanisms (different $\beta$ and $\mathrm{R}$ ) producing very similar $\sigma_{\mathrm{c}}$. It is not possible to identify the most likely failure mechanism - it appears rather indeterminate. 

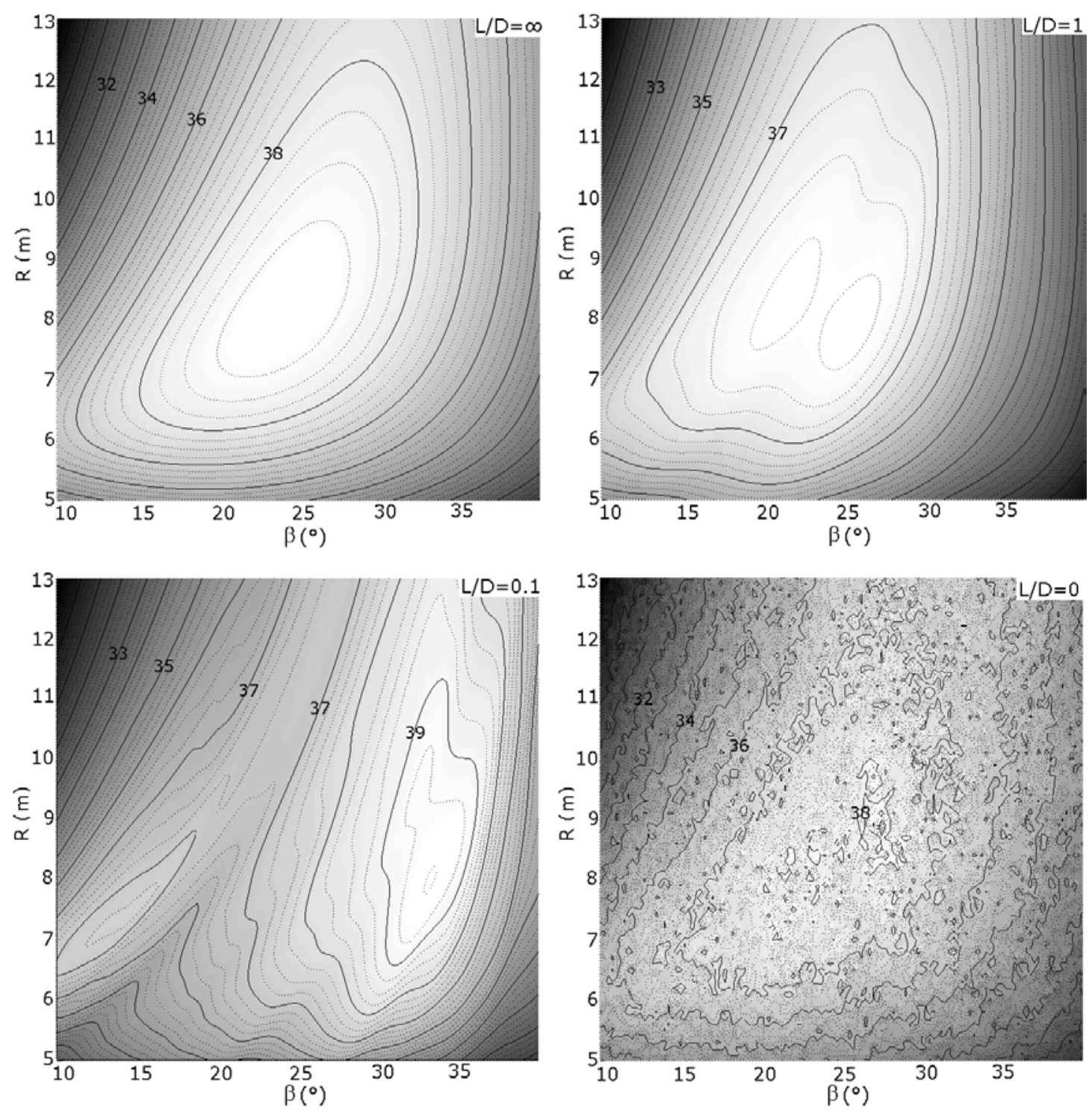

Figure 2. Response surfaces (in kPa) for several values of $L / D$

\section{MONTE-CARLO SAMPLING}

Several values of $\mathrm{L} / \mathrm{D}$ ranging from 0.05 to 2 are considered, as well as the two limit cases $\mathrm{L} / \mathrm{D}=0$ and $\mathrm{L} / \mathrm{D}=\infty$. For each case, a Monte-Carlo simulation is carried out using 10,000 samples. For each sample, the value of the global maximum $\sigma_{\mathrm{c}}$ is stored, as well as the corresponding parameters $\beta, \mathrm{R}, \mathrm{H}$, and $\mathrm{R}_{\mathrm{m}}$ (except for the case $\mathrm{L} / \mathrm{D}=0$, for which the location of the global maximum is indeterminate as may be seen from Fig. 2). Moreover, when a local maximum appears, the collapse pressure and the corresponding parameters are stored as well. It was found that, for all the values of $L / D$ and for the range of the samples examined, the parameters $H$ and $R_{m}$ of the global and local maximums were always equal to $0 \mathrm{~m}$ and $5 \mathrm{~m}$ respectively, which means that the spatial variability of $\varphi$ in a sand is not able to trigger collapses involving only a part of the tunnel face. Thus, only full-face failures are considered here, and the only varying parameters ( $\beta$ and $R$ ) are those related to the position of point $\mathrm{O}$ which represents the centre of rotation of the failure mechanism. 

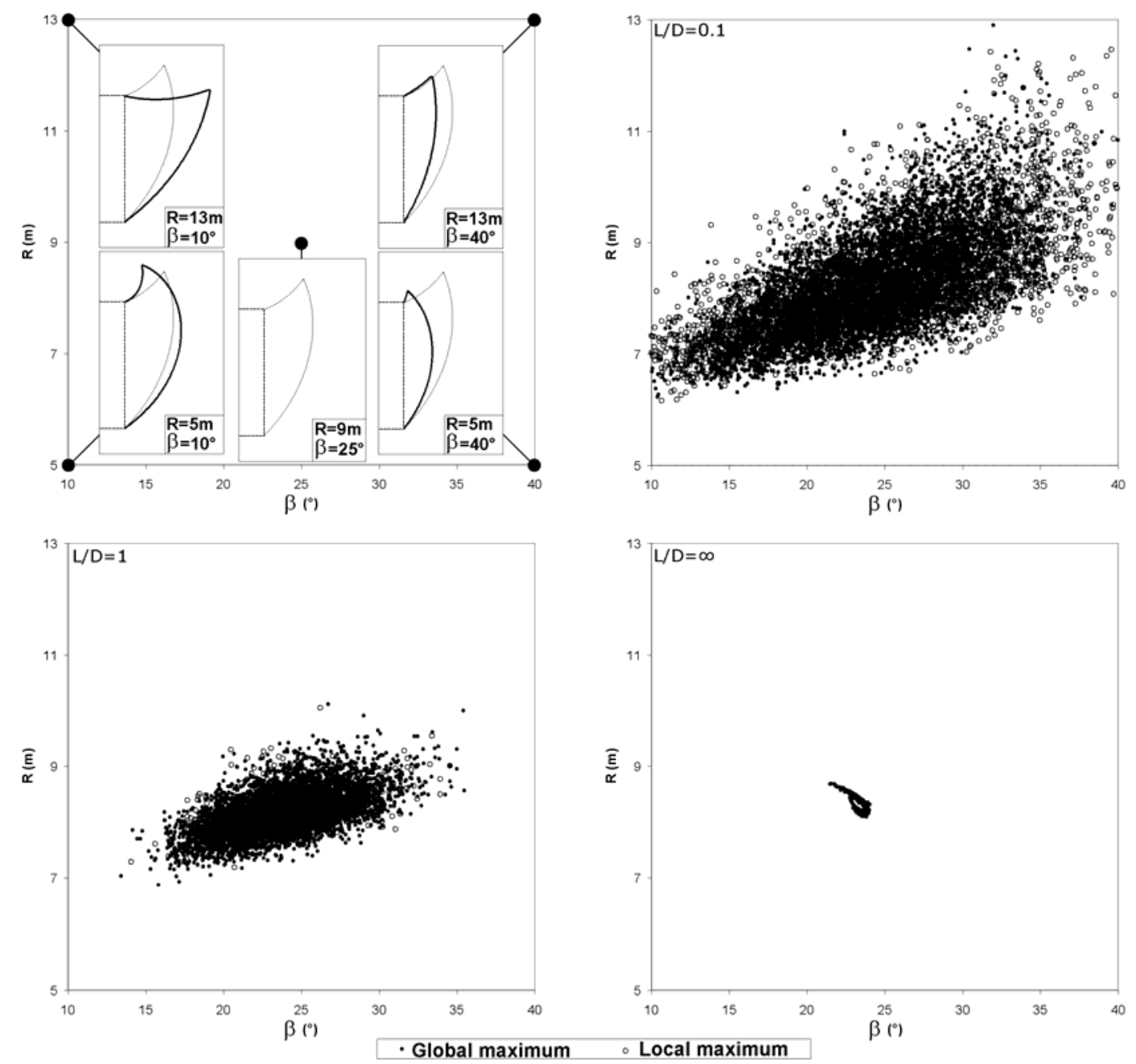

Figure 3. Positions of the global and local maximums of $\sigma_{c}$ as obtained from the 10,000 samples for various values of $L / D$

The positions in the $(\beta, \mathrm{R})$ plane of the global and local maximums as obtained from the different samples are plotted in Fig. 3. It clearly appears that a small value of L/D leads to a large cloud of maximums and thus to various collapse shapes. On the contrary, a large value of L/D leads to a small variability for the parameters $\beta$ and $\mathrm{R}$, and thus to a quite "standard" collapse mechanism. Notice that Fig. 3 also shows the critical positions of the failure mechanism for different values of $\beta$ and $\mathrm{R}$.

Fig. 4a shows the evolution of the coefficients of variation (COV) of $\beta, \mathrm{R}$, and $\sigma_{c}$ with L/D. The observation of Fig. 3 concerning the variability of $\beta$ and $R$ is confirmed by the values of the COV of $\beta$ and R given in Fig. 4a. Indeed, the COV of the two parameters $\beta$ and $\mathrm{R}$ are equal to $21 \%$ and $12 \%$ respectively for a very small scale of fluctuation $(\mathrm{L} / \mathrm{D}=0.05)$, and decrease to very small values $(1 \%$ and $0.5 \%$ respectively) for a very large scale of fluctuation $(\mathrm{L} / \mathrm{D}=\infty)$. On the other hand, the $\mathrm{COV}$ of $\sigma_{\mathrm{c}}$ increases with $\mathrm{L} / \mathrm{D}$, from $4 \%$ (for $\mathrm{L} / \mathrm{D}=0.05$ ) to $16 \%$ (for $\mathrm{L} / \mathrm{D}=\infty$ ). The simple assumption $L / D=\infty$ is therefore conservative in terms of the variability of $\sigma_{c}$. It is interesting that $\mathrm{L} / \mathrm{D}=\infty$ produces almost the same mechanism but exhibits a large variability in $\sigma_{\mathrm{c}}$. On the contrary, $L / D=0$ produces a large variety of failure mechanisms, but exhibits almost the same $\sigma_{\mathrm{c}}$. At first glance, these results seem rather 
counter-intuitive. However, the fact that a smaller variability of $\sigma_{\mathrm{c}}$ was obtained for small L/D values may be explained as follows: for a very small autocorrelation distance, the zone involved by the possible failure mechanism will have average values of the friction angle close to the mean value of the random field because of the large number of high and small values of the friction angle. This leads to close values of the collapse pressure and thus to a smaller variability in this pressure. On the other hand, the fact that a great variability in $\beta$ and $\mathrm{R}$ was obtained for a small autocorrelation distance may be related to the fact that several paths of the failure mechanism would be possible due to the quick changes in the values of the friction angle in this case.
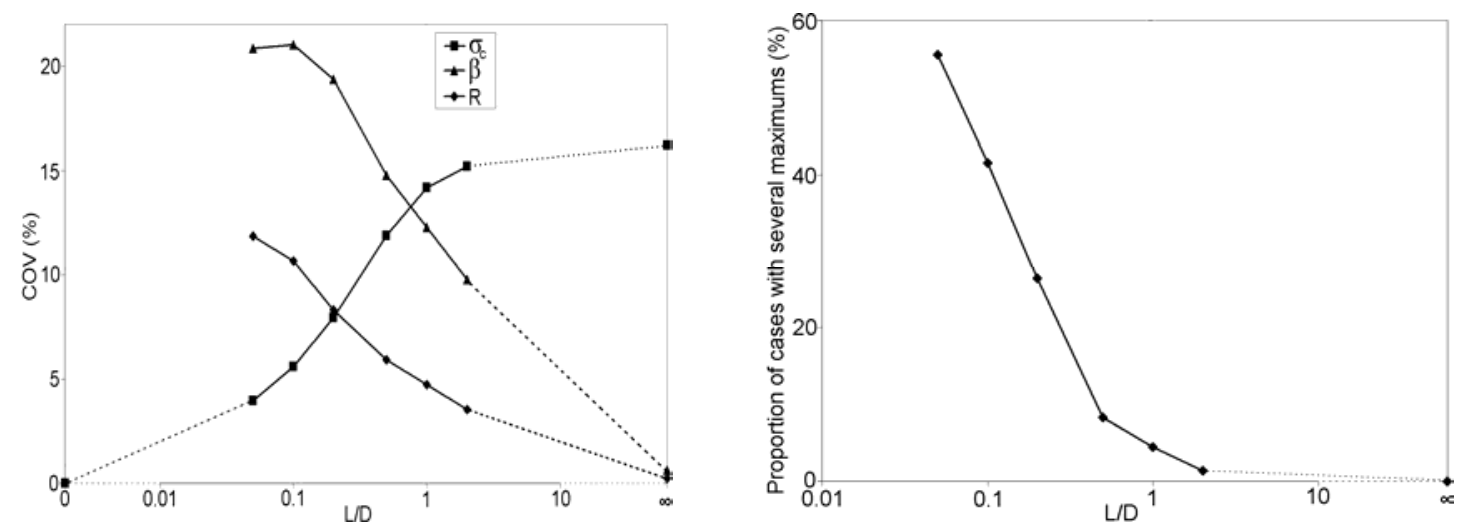

Figure 4. a. Influence of $L / D$ on $C O V$ of $\sigma_{c}, \beta_{c}$, and $R_{c}$; $b$. Influence of $L / D$ on the proportion of realizations leading to several maximums
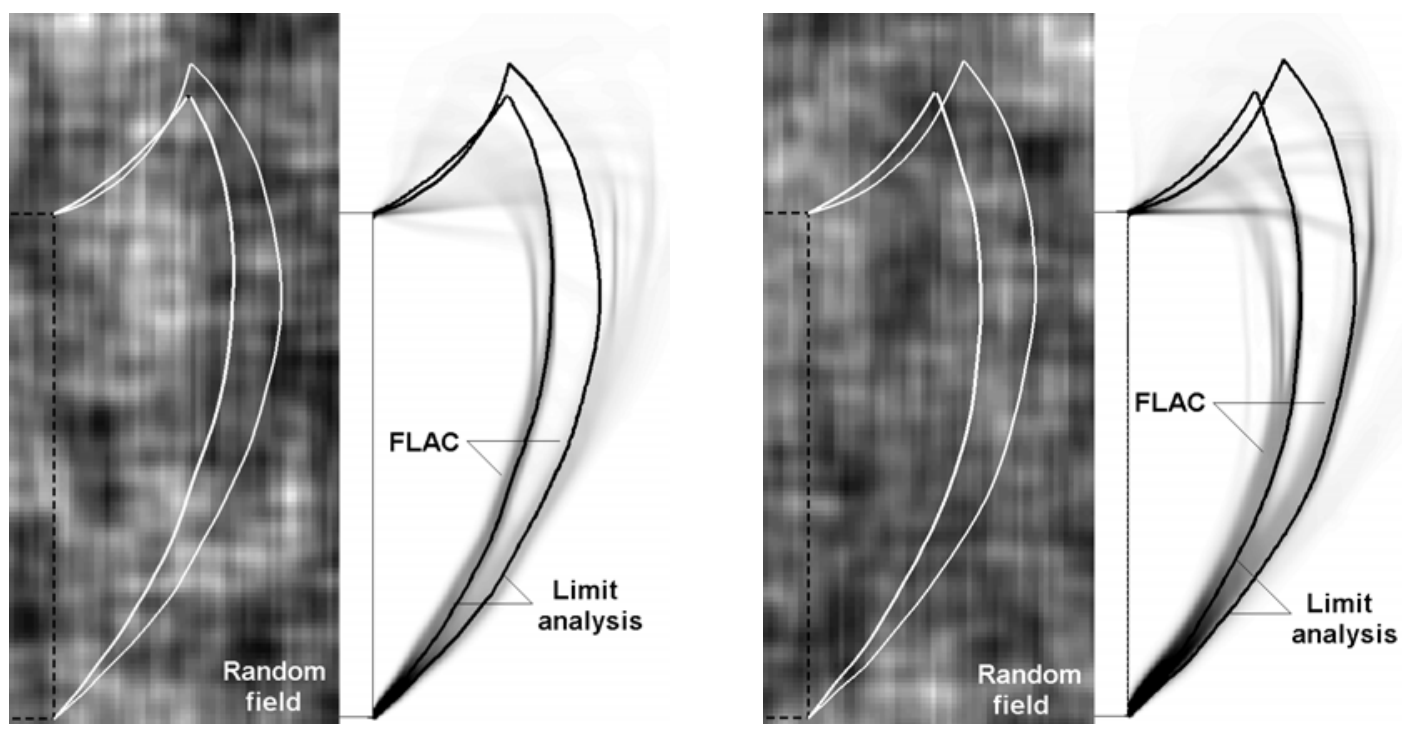

\section{Figure 5. Comparison between limit analysis and FLAC for two examples of realizations leading to multiple failures.}

Fig. $4 \mathrm{~b}$ shows the impact of $\mathrm{L} / \mathrm{D}$ on the proportion of samples leading to several maximums. For $\mathrm{L} / \mathrm{D}=\infty$, this proportion is zero because homogeneous soils always lead to very regular response surfaces (Fig. 2). However, it appears that this proportion increases when $\mathrm{L} / \mathrm{D}$ decreases and can reach $55 \%$ for $\mathrm{L} / \mathrm{D}=0.05$. When several maximums exist, the global maximum may have a much larger value of $\sigma_{\mathrm{c}}$ 
than the local one. In such a case, one can assume that the local maximum has a limited importance and that the global maximum is the one corresponding to the real failure. However, Monte-Carlo results show that $3.8 \%$ of the samples for $\mathrm{L} / \mathrm{D}=1$ leads to two maximums with a relative difference smaller than $1 \%$ in terms of $\sigma_{\mathrm{c}}$. For $\mathrm{L} / \mathrm{D}=0.1$, this proportion reaches $18.5 \%$. In such a case, when two maximums have very close values of $\sigma_{c}$, it is very difficult to discern which mechanism or a combined mechanism will be realized in reality. On Fig. 5, one can observe the collapse mechanisms obtained for two samples exhibiting such a "multiple" failure, for $\mathrm{L} / \mathrm{D}=0.1$. Two failure mechanisms corresponding to the two close maximums of $\sigma_{\mathrm{c}}$ are plotted for each sample. In order to verify the obtained failure patterns, the software FLAC is used to set up a numerical model (Fig. 6a). FLAC is a commercial program based on a lagrangian finite differences resolution scheme. The critical collapse pressure $\sigma_{\mathrm{c}}$ is found by a bisection method described in Mollon et al. 2011, and the failure pattern is obtained by applying $\sigma_{\mathrm{c}}-0.3 \mathrm{kPa}$ to the tunnel face. The advantage of the FLAC model when compared to the proposed limit analysis model is that it does not imply any assumption of the failure velocity field. It is therefore a convenient verification tool, despite its expensive computational cost. The failure patterns obtained by FLAC are provided as well in Fig. 5, and show a good agreement with the limit analysis results. The spatial variability of $\varphi$ in sands is therefore able to trigger qualitatively different and more complex failure mechanisms involving several concurrent slip-lines, which do not appear in homogeneous soils.
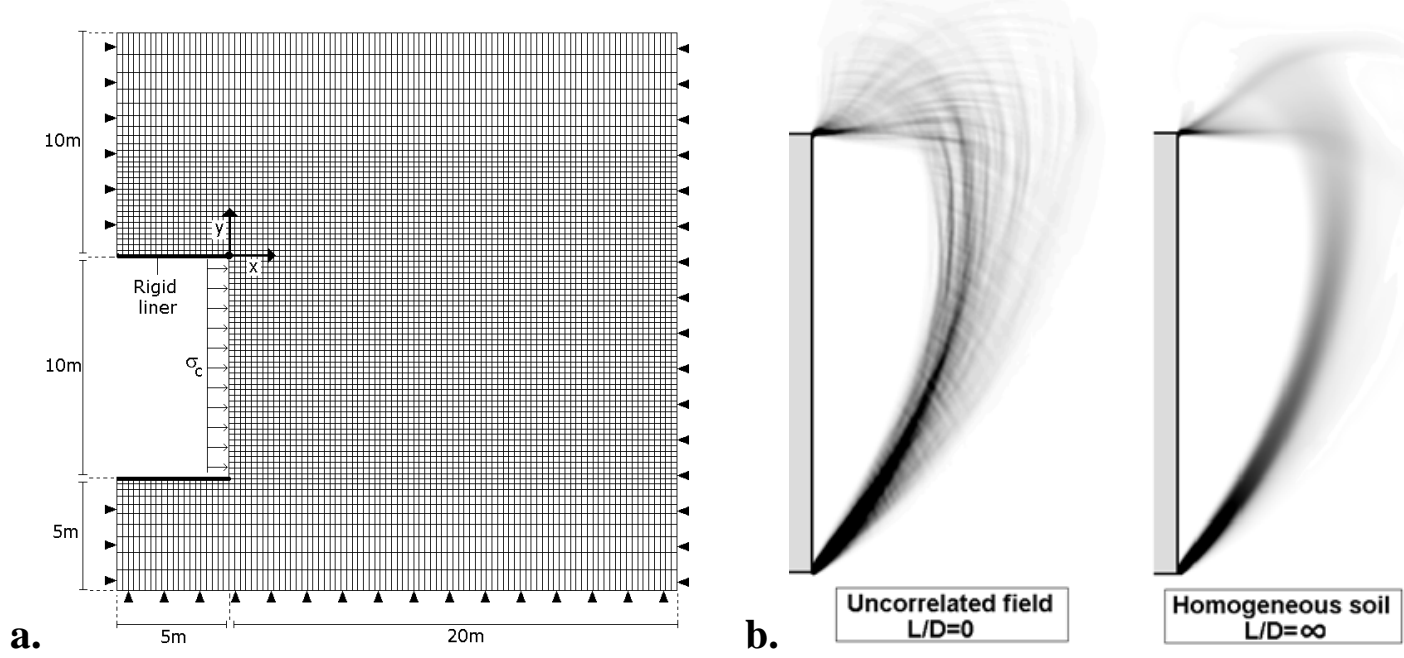

Figure 6. a. Flac Model; b. Failure shapes for the two limit cases

\section{STUDY OF THE LIMIT CASE L/D=0}

When observing the response surface of the limit case $\mathrm{L} / \mathrm{D}=0$ (Fig. 2), it is difficult to assess if the number of maximums is very large (if one considers all the irregularities of the response surface as local maximums) or if there is only one maximum (if one considers that those irregularities are irrelevant and that the surface has to be smoothed). The large number of local maximums would lead to a large number of concurrent collapse mechanisms. On the other hand, the irregularities of the surface could potentially be interpreted as numerical artifacts/noises superposed to an "actual" smooth surface, which would lead to a single mechanism and a failure 
close to the ones observed in homogeneous soils. On Fig. 6b, one can observe the failure shapes obtained by the FLAC model for the two limit cases $\mathrm{L} / \mathrm{D}=0$ and $\mathrm{L} / \mathrm{D}=\infty$. According to these results, it seems that the numerous local maximums observed on the response surface of the case $L / D=0$ are physically meaningful, since the failure apparently involves a large number of slip lines. Thus, FLAC and limit analysis results seem to be consistent. However, it could mean that both approaches are wrong in the sense that the approximate method of representing an uncorrelated field used in this study may be inadequate. In such a case, a smoothing process applied to the response surface may be acceptable, and the maximum may be unique. This would imply the existence of a "critical" autocorrelation length, for which the proportion of multiple failures would be maximum at an intermediate autocorrelation length between the two limits, $\mathrm{L} / \mathrm{D}=0$ and $\mathrm{L} / \mathrm{D}=\infty$. Nevertheless, the existence of very small autocorrelation lengths may be academic because these scales of fluctuation are usually smoothed by the common geotechnical experimental laboratory or field devices, which deal with meso-scale samples and meso-scale averaged parameters. The physical interpretation of classical parameters such as the friction angle at very small scales is currently tenuous. These important issues are left for further studies.

\section{CONCLUSION}

A two-dimensional limit analysis mechanism is used in this paper to study the impact of the spatial variability of the friction angle of sands on the stability of a pressurized tunnel face. A Monte-Carlo method is used to carry out the stochastic simulations, and evaluate the impact of the autocorrelation length of the random field. It appears that the heterogeneities of the sand are not able to trigger partial failures (i.e. failures involving only a part of the tunnel face). A small autocorrelation length (with respect to the tunnel diameter) leads to a wide variety of collapse mechanisms, but reduces the dispersion of the critical collapse pressure. On the contrary, a large autocorrelation length leads to quite "standard" unique failure mechanism, but increases the coefficient of variation of the collapse pressure. The assumption of a homogeneous soil with a large autocorrelation distance is therefore conservative in terms of the variability of the critical collapse pressure. Moreover, it appears that small autocorrelation lengths are able to trigger multiple failures. This emerging phenomenon could not appear in homogeneous soils, and emphasises the practical interest of stability studies involving spatially variable soils.

\section{REFERENCES}

Mollon, G., Phoon, K.-K., Dias, D., and Soubra, A.-H. (2011), "Validation of a new 2D failure mechanism for the stability analysis of a pressurized tunnel face in a spatially varying sand", J. of Engrg. Mech, ASCE, in press.

Phoon, K.K., Huang, H.W., and Quek, S.T. (2005). "Simulation of strongly nonGaussian processes using Karhunen-Loeve expansion.” Prob. Engrg. Mech., 20 (2), 188-198.

Sudret, B, and Der Kiureghian, A. (2000). "Stochastic finite element methods and reliability, a state of the art”, Report nUCB/SEMM-2000/08, November 2000, Dpt. of Civil and Environmental Engineering, Berkeley, California. 